\title{
Nuclear Magnetic Resonance Imaging of Skull Base Lesions
}

\author{
D.G. Potts and R.D. Zimmerman
}

\begin{abstract}
The basic principles of nuclear magnetic resonance (NMR) are reviewed and the appearances of some lesions of the skull base are discussed. NMR is a valuable diagnostic study for skull base lesions and it provides information that is not shown on the CT scan. The modern investigation of unusual skull base lesions should include both CT and NMR.
\end{abstract}

RÉSUME: La résonance magnétique nucléaire et les lésions de la base du crâne Nous passons en revue les principes de base de la résonance magnétique nucléaire (NMR) et décrivons l'image des lésions de la base du crâne. Cette approche radiologique est d'une aide précieuse au diagnostic de ces lésions de la base du crâne et permet d'obtenir de l'information non disponsible avec la tomodensitométrie. L'emploi des deux techniques pour l'étude des lésions inhabituelles de la base du crâne devrait s'avérer la norme moderne.

Can. J. Neurol. Sci. 1985; 12:327-331

Nuclear magnetic resonance (NMR) imaging techniques may be used to study certain atomic nuclei such as hydrogen, sodium and phosphorus that have odd numbers of protons or neutrons. ${ }^{\prime}$ These nuclei are positively charged and they spin at a high speed (considerably higher than radio frequencies). Most NMR clinical images provide information about the hydrogen nucleus or proton. When a proton is placed in a powerful magnetic field it tends to align with the field but because of thermal motion of the atoms the proton does not spin around a stationary axis parallel to the magnetic field; it tends to "precess" or wobble like a spinning top that has been pushed of the vertical position. The precession frequency is higher when the proton is in a more powerful magnetic field and medical NMR scanners have a magnetic field powerful enough to cause the protons to precess at a frequency in the radiofrequency range.

In clinical NMR scanners the uniform primary magnetic field of the magnet is modified by "field gradient coils" that result in different field strengths (and therefore different proton precession frequencies) from one region to another.

NMR scanners are equipped with a radiofrequency transmitter and a transmitter coil (or antenna) near the patient. When the transmitter is turned on and emits a signal at a certain frequency those protons with the same precession frequency will absorb energy. If the radio transmitter is then turned off, the protons that have absorbed energy will emit a radio signal of the same frequency and this signal can be detected by means of a special radio receiver. The source of the signal may be deduced from the frequency of the emitted signal and this information is used to generate the image. The initial amplitude of the signal is related to the proton density. Thus, images that show the distribution of protons (or hydrogen) may be formed by measuring the initial amplitude of the radio signal.

Following radio stimulation of the protons the emitted signal falls off at a rate and with a pattern that is related to the characteristics of the tissues in which the protons are situated. The shape of the diminishing curve of signal amplitude may be separated into two separate components or "relaxation times". The first of these (the "Tl" relaxation time) is determined by the mechanical rigidity with which the proton is held in relation to its surroundings: $\mathrm{Tl}$ is therefore very long for solids and shorter for liquids. The second relaxation time (T2) gives an assessment of the uniformity or non-uniformity of the environments of the protons. Pure (uniform) water has a longer T2 time than impure liquids.

Finally these images give information about flow. When clinical images are being generated the transmitter is turned on for a certain time and then the signal amplitude may be measured after a delay (often approximately one second). This delay before observing the signal strength may result in a "signal washout" effect due to fast moving blood in arteries or veins.

Proton density images are rarely used today for clinical diagnosis. Pure T1 or T2 images cannot easily be produced as primary images today, but by appropriate stimulation ("pulsing") strategies the images produced may provide mainly $\mathrm{T} 1$ information or mainly T2 information (T1 or T2 "weighted" information). ${ }^{2.3}$ 


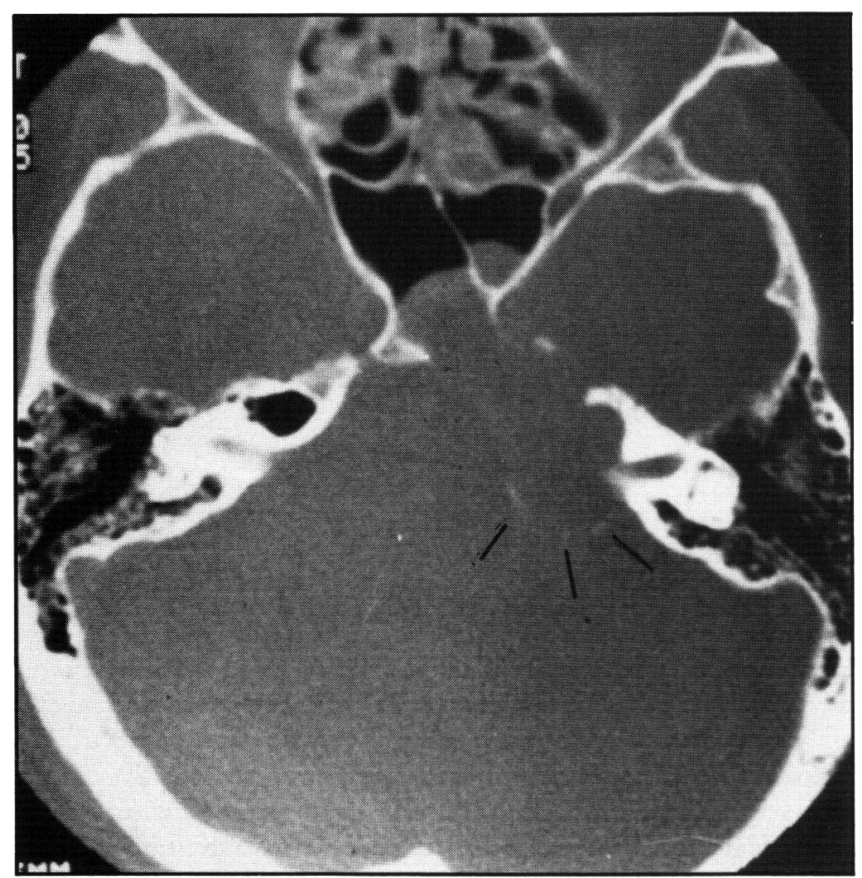

A

Figure I - Epidermoid in the apex of the petrous temporal bone.

A) CT scan showing a large bony defect in the apex of the left petrous temporal bone and a soft tissue mass projecting into the sphenoid sinus.

B) NMR scan showing the high signal caused by this fat-containing tumour.

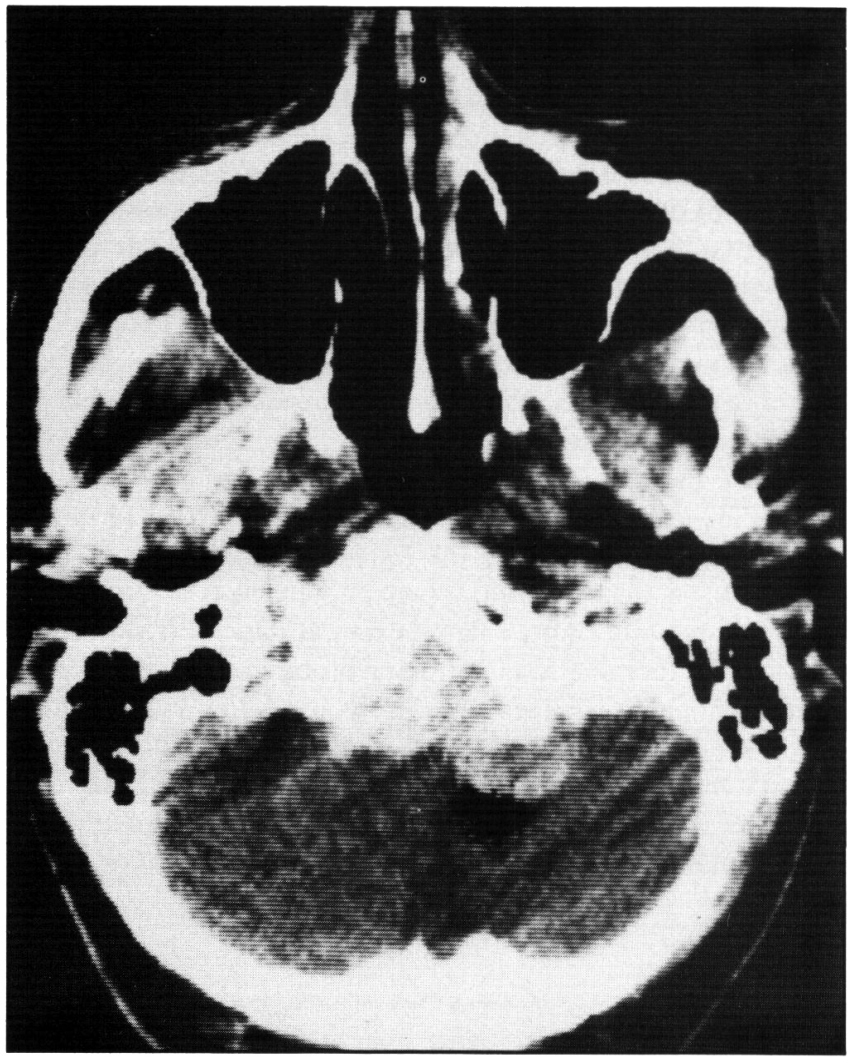

A

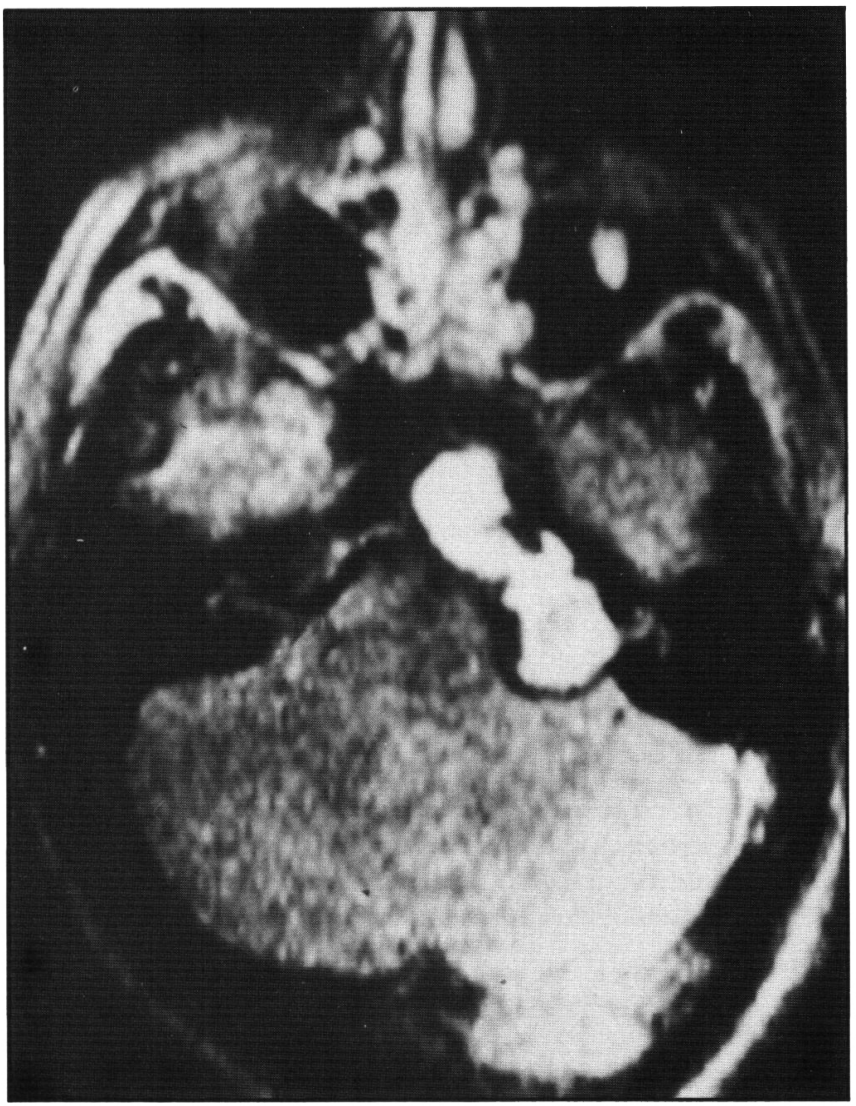

B

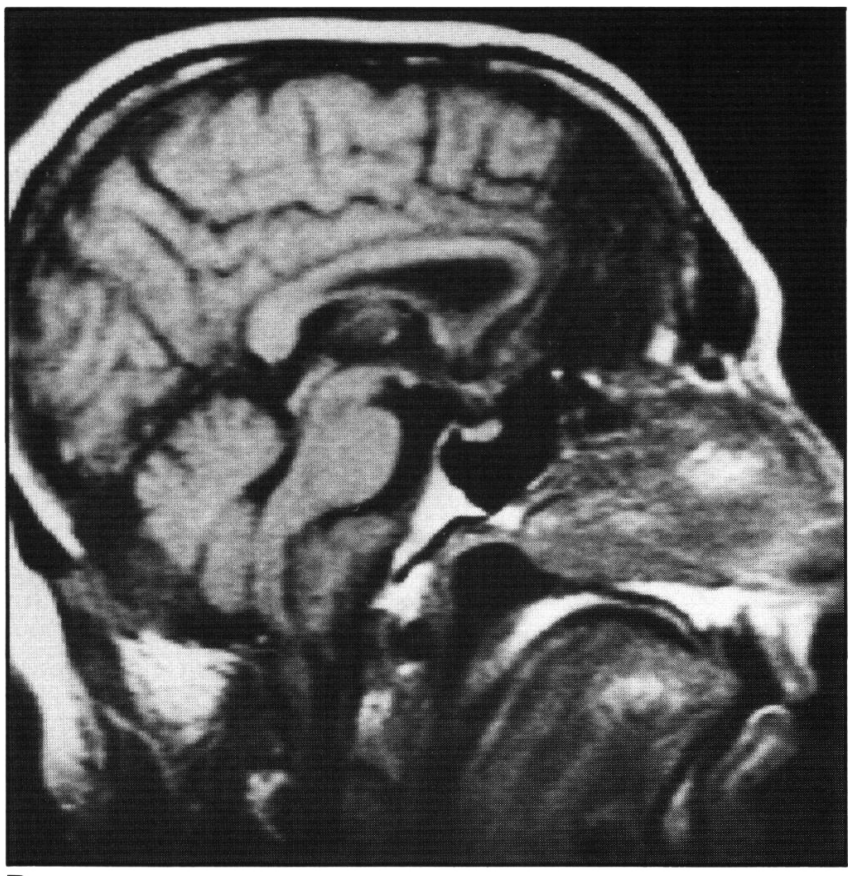

$\underline{B}$

Figure 2 - Clivus meningioma

A) CT scan showing the enhancing tumour extending behind the clivus.

B) NMR scan showing the tumour displacing the brainstem posteriorly. 


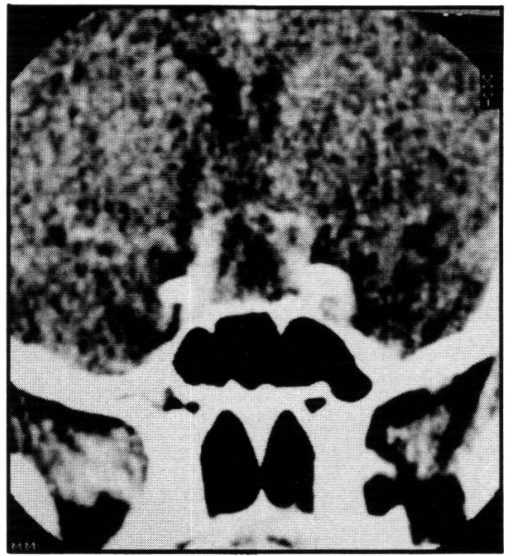

A

Figure 3 - Pituitary adenoma with cystic changes.

A) CT scan (following intravenous contrast) showing the tumour extending into the suprasellar region.

B) NMR scan, coronal view clearly shows the large tumour.

C) Postoperative scan showing almost complete removal of the tumour. Note the optic nerve passing downward and forward.
T1 weighted images provide excellent anatomical detail in the brain, with good gray-white matter discrimination, and with the cerebrospinal fluid appearing blacker than the surrounding brain. T2 weighted images will show more "noise" and less anatomical detail, and with the cerebrospinal fluid whiter than the surrounding brain.

The actual T1 and T2 values of biological tissues will depend on the primary magnet strength. Magnets in scanners used for medical diagnosis range from 0.15 tesla to 2.0 tesla. While the assessment of the relative merits of magnets of different strengths is controversial, there seems to be some evidence that weaker magnets (about 0.3 tesla) give better $\mathrm{T} 1$ discrimination, while more powerful magnets give better $\mathrm{T} 2$ discrimination. Most acquired lesions in the brain (tumours, inflammation, infarcts, contusions, and demyelination) cause more prominent $\mathrm{T} 2$ changes and they tend to be shown more clearly on an imaging unit with a more powerful magnet.

While this simple description of the principles of NMR imaging is somewhat oversimplified, it provides the essential information necessary for the rational application of the technique for medical diagnosis.

\section{Advantages of NMR}

Compared with computed tomography (CT scan), NMR images have some significant advantages. No radiation is required for the production of NMR images and adverse effects are very unlikely provided the patient does not have a cardiac pacemaker or ferrous material (for example, an aneurysm clip) within the cranium. While CT images near the skull base show streak artefacts caused by beam hardening, NMR images show more clearly the soft tissues close to the skull base because the images are free of streak artefacts and dense bone gives a low signal intensity. Major arteries and veins are clearly shown in NMR images as regions of low intensity signals indicating normal blood flow. NMR images may be readily obtained in any plane. Lateral sections may be particularly valuable for the demonstration of lesions of the skull base. Some tissues (e.g. fat) and some lesions (e.g. demyelination or edema) are demonstrated more effectively by NMR.

\section{Disadvantages of NMR}

Abnormalities of compact bone are usually not well seen in NMR images while they can be clearly demonstrated in CT sections. It may therefore be an advantage to combine the information on a CT scan with that on the NMR scan. Because NMR sections require from 30 seconds to 30 minutes (depending on the information desired) they are more likely to be degraded by patient motion than $\mathrm{CT}$ scans which can be obtained in one to two seconds with a modern scanner.

\section{Normal Skull Base}

The compact bone of the skull base gives a very low signal while cancellous bone containing marrow (in the clivus, or the diploic region of the cranial vault) gives a higher signal. The larger cranial nerves in the region of the skull base may be seen in NMR images as narrow bands of higher signal intensity than the surrounding structures. ${ }^{4}$ Blood vessels are clearly seen and the images indicate whether they have normal blood flow. The brainstem and upper spinal cord are usually seen more clearly on NMR images than on CT sections. Although fractures of the skull base are not usually seen on NMR sections, blood in the mastoid air cells or paranasal sinuses may indicate the presence of a fracture. The signal from the blood increases in intensity for the first few days after injury and the presence of blood is most easily recognized at this time.

The most commonly used imaging technique is the "spinecho" technique. In these images fat and bone marrow give the brightest signals, and compact bone and air give the weakest signals. Generally tissues that give a bright (strong) NMR signal have a short T1, a long T2, or both. Tissues that have a black (weak) signal have a long T1, a short T2, or both.

\section{Abnormal Skull Base}

Experience in the use of NMR for the diagnosis of skull base lesions is still accumulating. However, it is already clear that NMR has important advantages. While CT scan images are obtained with a fairly standard imaging strategy and provide information about $x$-ray attenuation values, NMR images may be formed with a wide range of imaging strategies and provide 


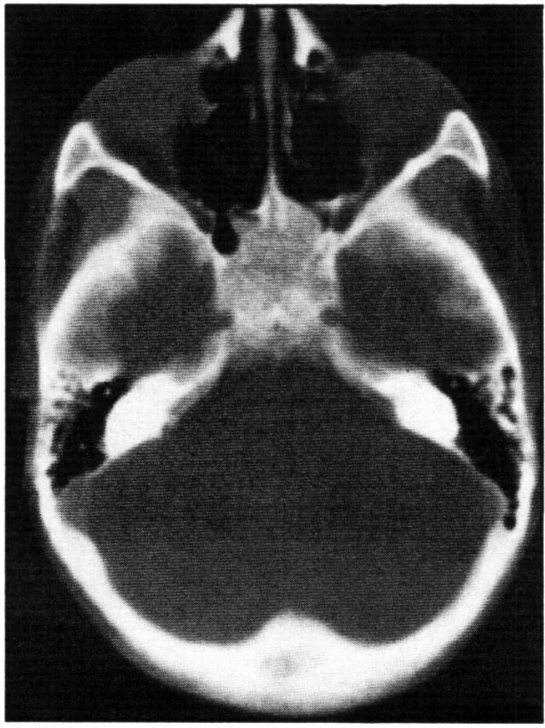

A
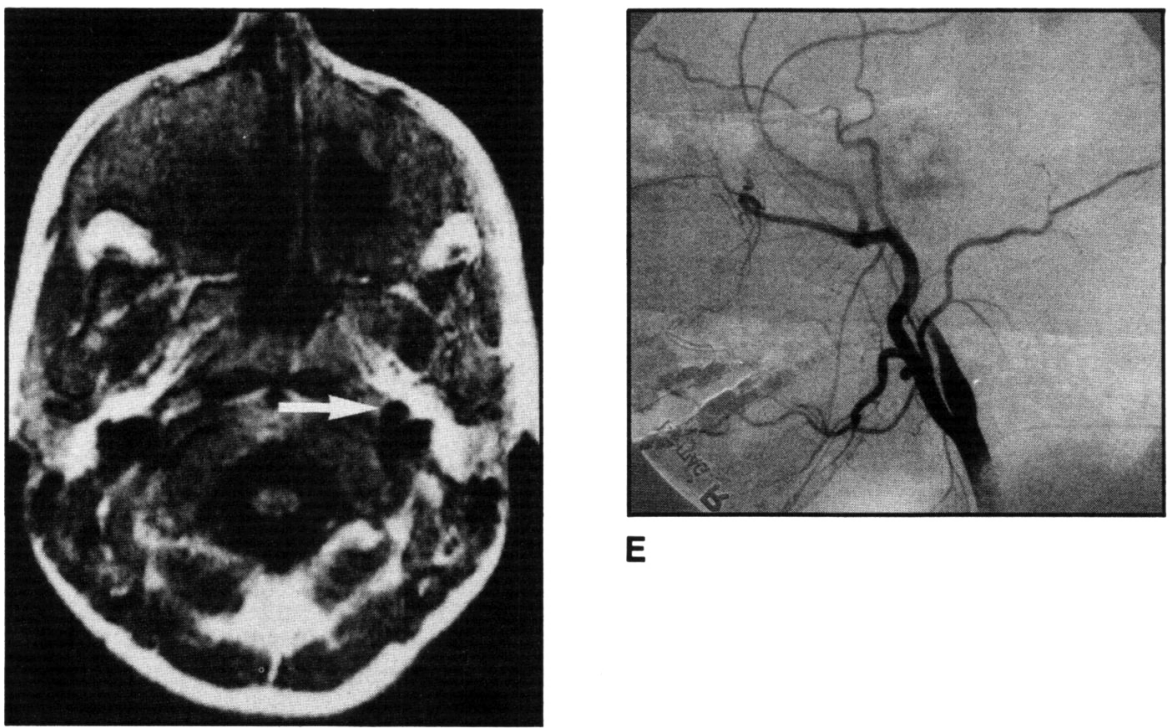

E

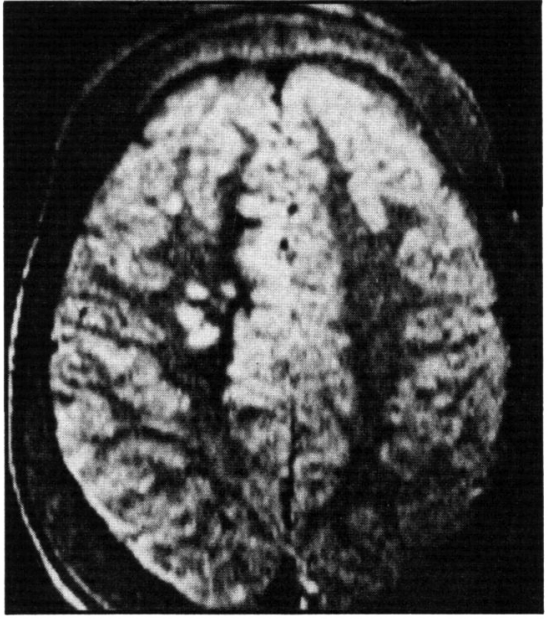

B

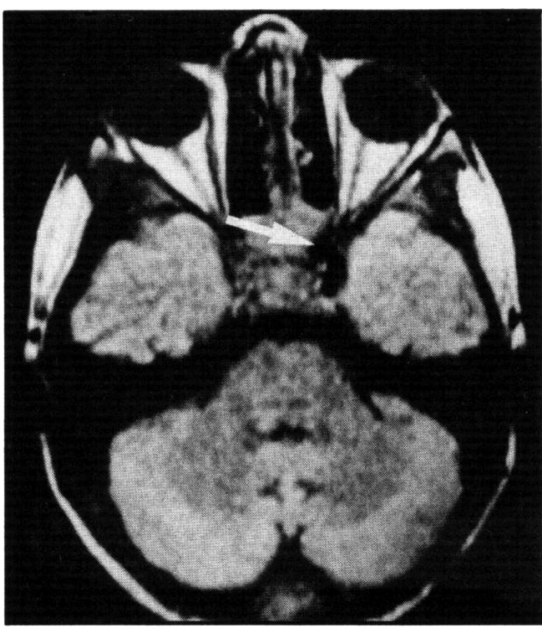

C

D

Figure 4 - Fifteen-year-old boy with thalassemia major and right carotid occlusion causing left hemiplegia.

A) CT scan showing expansion of the sphenoid body by marrow hypertrophy. The carotid artery grooves are clearly seen. Evidence of bony hypertrophy is also seen in the skull anterior to the middle cranial fossa.

B) NMR scan showing nodular regions of increased signal due to an infarct in the right frontoparietal region superiorly.

C) NMR scan through the body of the sphenoid showing flow in the left internal carotid artery (arrow) but no flow in the right internal carotid artery.

D) NMR scan through the inferior aspect of the skull base showing flow in both jugular veins, and the left internal carotid artery (arrow), but no flow in the right internal carotid artery.

E) Right carotid angiogram showing occlusion of the right internal carotid artery and thrombosis extending down the cervical part of the artery.

The occlusion was considered to be due to marrow overgrowth compressing the internal carotid artery in the parasellar region.

at least four different types of information: proton density, $\mathrm{Tl}$, T2 and flow. In fact, most NMR images represent more than one type of information. Changes in the imaging strategy may cause dramatic changes in the appearance of normal structures or abnormal lesions.

\section{Clinical Examples}

Developmental Tumours These tumours are usually displayed very clearly by NMR. Fat has a short T1 and gives a bright signal and therefore congenital tumours containing fat show a bright signal (Figure I). Calcium gives a very weak signal and is not demonstrated by NMR unless it is very extensive, when it appears as a region of low intensity signal.

Meningiomas Meningiomas usually give NMR signals that are not very different from the adjacent brain but the presence of a meningioma of the skull base may be clearly shown by the displacement of adjacent normal structures (Figure 2). ${ }^{5}$

Pituitary Tumours Pituitary adenomas are well shown by NMR if they are at least $1 \mathrm{~cm}$ in size. ${ }^{6}$ Smaller tumours may not be as reliably demonstrated as they are by $\mathrm{CT}$. Median sagittal 
NMR views are particularly useful and it is usually possible to demonstrate the optic nerves and chiasm (Figure 3).

Arterial Occlusion Thrombosis of medium-sized arteries or veins may be diagnosed when the absence of the signal "washout" effect due to flow is noted (Figure 4). Displaced or compressed arteries are also shown by NMR.

\section{REFERENCES}

1. Newton TH and Potts DG. Modern Neuroradiology, Vol. 2. San Anselmo, California: Clavadel Press, 1983.
2. Kjos BD, Ehman RL and Brant-Zawadzki. Reproducibility of T1 and $T 2$ relaxation times calculated from routine $M R$ imaging sequences: Phantom study. Am J Neuroradiol 1985; 6:277-283.

3. Kneeland JB, Knowles RJR and Cahill PT. Multi-section multi-echo pulse magnetic resonance techniques: Optimization in a clinical setting. Radiology 1985; 155:159-162.

4. New PFJ, Bachow TB, Wismer GL et al. MR imaging of the acoustic nerves and small acoustic neuromas at $0.6 \mathrm{~T}$ : Prospective study. Am J Neuroradiol 1985; 6:165-170.

5. Zimmerman RD, Fleming CA, Saint-Louis LA et al. Magnetic resonance imaging of meningiomas. Am J Neuroradiol 1985; 6:149-157.

6. Daniels DL, Pech P, Mark L et al. Magnetic resonance imaging of the cavernous sinus. Am J Neuroradiol 1985; 6:187-192. 